\title{
Acciones de mejora en el proceso editorial científico del Journal Boliviano de Ciencias: estudio de caso
}

\author{
ROCHA, Joaquin Humberto Aquino ${ }^{1^{*}}$ \\ MONTAÑO-BAUTISTA, Edson Gastón ${ }^{2 * *}$ \\ IÑIGUEZ-CALVETI, Daysi Lidia ${ }^{3 * * *}$ \\ ${ }^{1,2,3}$ Universidad Privada del Valle - Bolivia \\ ORCID ID: https://orcid.org/0000-0002-3383-6379* \\ ORCID ID: https://orcid.org/0000-0002-7105-9072** \\ ORCID ID: https://orcid.org/0000-0001-8101-0427 $7^{\text {**** }}$
}

\begin{abstract}
Resumen
El objetivo del presente estudio es determinar acciones de mejora para el proceso editorial del Journal Boliviano de Ciencias a través de la opinión de los autores. La metodología consistió en la aplicación de un cuestionario en línea a los autores de los últimos tres años (2017, 2018 y 2019), y del último semestre del 2020. Los resultados muestran que la revista debe mejorar en la visibilidad internacional, indexación y rapidez de la revisión por pares, principalmente. La mayoría de los autores está conforme con el proceso editorial y volvería a publicar nuevamente en la revista. Se debe trabajar en mejorar la calidad general de la revista para lograr indexación e índices académicos.

Palabras clave: Encuesta a autores. Escala de Likert. Publicación científica.
\end{abstract}

\section{Improvement actions in the scientific editorial process of Journal Boliviano de Ciencias: case study}

\begin{abstract}
The objective of this study is to determine improvement actions for the editorial process of Journal Boliviano de Ciencias through the opinion of the authors. The methodology consisted in the application of an online questionnaire to the authors of the last three years (2017, 2018 and 2019), and the last semester of 2020. The results show that the journal must improve in international visibility, indexing and speed of peer review, mainly. Most of the authors are satisfied with the editorial process and would re-publish in the journal. Work should be done to improve the general quality of the journal to achieve indexing.

Keywords: Survey to authors. Likert scale. Scientific publication.
\end{abstract}

\section{Ações de melhoria no processo editorial científico no Journal Boliviano de Ciencias: estudo de caso}

\begin{abstract}
Resumo
O objetivo deste estudo é determinar ações de melhoria para o processo editorial da Revista Boliviana de Ciências por meio da opinião dos autores. A metodologia consistiu na aplicação de um questionário online aos autores dos últimos três anos (2017, 2018 e 2019), e do último semestre de 2020. Os resultados mostram que a revista deve melhorar em visibilidade internacional, indexação e agilidade de revisão por pares, principalmente. A maioria dos autores está satisfeita com o processo editorial e publicaria novamente na revista. Deve-se trabalhar para melhorar a qualidade geral da revista para obter indexação e índices acadêmicos.
\end{abstract}

Palavras-chave: Questionário para autores. Escala de Likert. Publicação científica. 


\section{Introducción}

El descubrimiento de conocimientos científicos genera numerosos beneficios para la humanidad, siendo el objetivo principal de la ciencia. La comunicación y publicación de resultados de una investigación se realiza a través de artículos en revistas científicas, sistema que, desde su implementación, no ha cambiado sustancialmente (VRANA, 2018).

Aun así, existen desafíos para mejorar la calidad de las revistas científicas. Es necesaria la generación de acciones de mejora desde la gestión de revisión por pares y el proceso editorial de la revista, con el fin de reducir errores y comportamientos poco éticos (HAUSMANN; MURPHY, 2016). Considerando que, tanto las revistas pequeñas y nuevas enfrentan los mismos desafíos que aquellas consolidadas e importantes (MARGALIDA; COLOMER, 2016).

El Journal Boliviano de Ciencias (JBC) (ISSN: 2075-8936 y e-ISSN: 2075-8944) es la revista científica de la Facultad de Tecnología de la Universidad Privada del Valle, que comprende el área de las ingenierías, principalmente. Su difusión es nacional y de forma impresa, aunque en los últimos años se ha realizado una mayor abertura por la difusión digital. Dentro de esta línea, se busca la mejora de la calidad del JBC para lograr indexaciones internacionales e índices académicos.

El presente artículo tiene como objetivo determinar las acciones de mejora más importantes para el proceso editorial de revistas científicas, específicamente del JBC, a través de la opinión de los autores de los últimos años. Retroalimentación que, pueda generar lineamientos para ser implementados a fin de aumentar la calidad general del JBC.

\section{Metodología}

Para el presente estudio se realizó un cuestionario Google Forms, enviado a los autores que publicaron en el JBC durante los últimos tres años (2017, 2018 y 2019) y el último semestre del 2020. Se contabilizaron 96 autores durante el periodo estudiado, pero sólo 62 (64.58\%) respondieron a la encuesta.

En la primera parte del cuestionario, se consultó acerca de las características más importantes al momento de elegir una revista científica para publicar. Posteriormente, se propusieron algunas acciones de mejora para el proceso editorial del JBC, donde los autores señalaron las más importantes. Para esta primera parte se utilizó la escala de Likert. En la segunda parte del cuestionario, se consultó acerca de su conformidad y calificación (1 al 10) del proceso editorial y, finalmente, si volverían a publicar en el JBC. 
Para el análisis de resultados se utilizó el Índice de Importancia Relativa (IIR). El IIR fue calculado para la primera parte del cuestionario. En la segunda parte se realizó un análisis estadístico descriptivo.

La escala de Likert es la suma de todas las respuestas de cada clasificación. Utilizando cinco niveles de respuesta: cinco - muy relevante (MR), cuatro - relevante $(\mathrm{R})$, tres - regular relevante (RR), dos - poco relevante (PR) y uno - no relevante (NR).

El IIR se calcula mediante la ecuación (1). El numerador es la sumatoria del producto entre el valor de cada clasificación y su número de respuestas $\left(\sum x_{i} y_{i}\right)$. El denominador es el número total de respuestas de todas las clasificaciones $\left(\sum x_{i}\right)$. Es importante considerar que, si el IIR está cercano a cinco, mayor importancia adquiere.

$$
I I R=\frac{\sum x_{i} y_{i}}{\sum x_{i}}
$$

\section{Resultados y discusión}

La Tabla 1 presenta las consideraciones de los autores en la elección de la revista científica. Se puede observar que la Indexación $(4,48)$ es la característica más importante seguida del Factor de Impacto $(4,32)$ y del Tiempo de publicación $(4,13)$. Mientras que el Idioma, Lugar y Normas editoriales son considerados como los menos importantes.

Tabla 1 - Características de elección de revista científica (autores)

\begin{tabular}{l|c|c|c|c|c|c|c}
\hline Características & $\begin{array}{c}\text { MR } \\
(\mathbf{5})\end{array}$ & $\begin{array}{c}\mathbf{R} \\
\mathbf{( 4 )}\end{array}$ & $\begin{array}{c}\text { RR } \\
(\mathbf{3})\end{array}$ & $\begin{array}{c}\text { PR } \\
(\mathbf{2})\end{array}$ & $\begin{array}{c}\mathbf{N R} \\
(\mathbf{1})\end{array}$ & IIR & Posición \\
\hline Indexación & 38 & 16 & 8 & 0 & 0 & 4,48 & 1 \\
\hline Factor de impacto & 30 & 24 & 6 & 2 & 0 & 4,32 & 2 \\
\hline Tiempo de publicación & 26 & 22 & 10 & 4 & 0 & 4,13 & 3 \\
\hline Tipo de arbitraje & 23 & 21 & 11 & 6 & 1 & 3,95 & 4 \\
\hline Tasa de aceptación/rechazo & 18 & 21 & 19 & 4 & 0 & 3,85 & 5 \\
\hline Idioma & 17 & 19 & 15 & 8 & 3 & 3,63 & 6 \\
\hline Lugar & 16 & 15 & 18 & 7 & 6 & 3,45 & 7 \\
\hline Normas editoriales & 4 & 12 & 27 & 14 & 5 & 2,94 & 8 \\
\hline
\end{tabular}

Fuente: Elaboración del autor (2020).

Para las acciones de mejora, en la Tabla 2 se observa la importancia asignada por los autores. Se considera que la Visibilidad internacional $(4,21)$, Indexación $(4,15)$ y Rapidez en la revisión por pares $(4,10)$ son las acciones que se deben mejorar, primordialmente. El JBC está trabajando en las ediciones digitales para mayor visibilidad además de reformas para poder ser 
integrada a diferentes bases de datos académicas. La revisión por pares está entre tres a seis meses, periodo reportando en las revistas científicas; no obstante, se trata de reducir los tiempos entre recepción y aceptación. Estos aspectos son considerados esenciales para medir la calidad de una revista científica (XIE; WU; LI, 2019).

Tabla 2 - Acciones de mejora para el JBC (autores)

\begin{tabular}{l|c|c|c|c|c|c|c}
\hline Acciones de mejora & $\begin{array}{c}\text { MR } \\
(\mathbf{5})\end{array}$ & $\begin{array}{c}\text { R } \\
\mathbf{( 4 )}\end{array}$ & $\begin{array}{c}\text { RR } \\
(\mathbf{3})\end{array}$ & $\begin{array}{c}\text { PR } \\
(\mathbf{2})\end{array}$ & $\begin{array}{c}\text { NR } \\
(\mathbf{1})\end{array}$ & IIR & Posición \\
\hline Mayor visibilidad internacional & 29 & 17 & 16 & 0 & 0 & 4,21 & 1 \\
\hline Indexación a otras bases & 28 & 16 & 17 & 1 & 0 & 4,15 & 2 \\
\hline Rapidez en la revisión por pares & 20 & 28 & 14 & 0 & 0 & 4,10 & 3 \\
\hline Contenido & 27 & 14 & 13 & 8 & 0 & 3,97 & 4 \\
\hline Menor tiempo de procesamiento & 21 & 19 & 20 & 2 & 0 & 3,95 & 5 \\
\hline Aumentar editores de sección & 9 & 34 & 15 & 4 & 0 & 3,77 & 6 \\
\hline Aceptar otros tipos de artículos & 22 & 10 & 23 & 6 & 1 & 3,74 & 7 \\
\hline Formato de publicación & 6 & 28 & 12 & 14 & 2 & 3,35 & 8 \\
\hline
\end{tabular}

Fuente: Elaboración del autor (2020).

Respecto a las otras acciones de mejora se busca mejorar el contenido a través de la exigencia de trabajos originales e inéditos en el área de las ingenieras. Los editores de sección fueron considerados desde el 2018; actualmente se tienen editores de sección para Ingeniería Civil, Industrial, Alimentos, Petróleo y Electromecánica. En el corto plazo se pretende incluir para Ingeniería Biomédica y Electrónica. Desde el 2020, el JBC se apertura a la recepción de diferentes tipos de trabajos técnicos y cartas al editor, dando la posibilidad al reporte de resultado negativos (BESPALOV; STECKLER; SKOLNICK, 2019).

Las Figuras 1a y 1b muestran la conformidad del proceso editorial y la disposición de publicar nuevamente en el JBC por parte de los autores, respectivamente. El 79\% está conforme con el proceso editorial desde la recepción hasta la publicación del manuscrito y un $84 \%$ de los autores volvería a publicar nuevamente en el JBC.

Figura 1 - a) Conformidad y b) disposición de publicar nuevamente en el JBC

a)

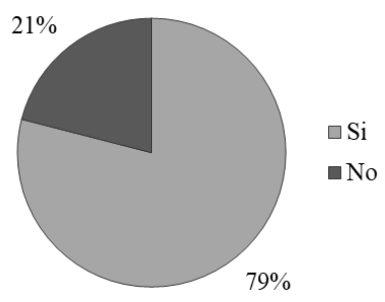

b)

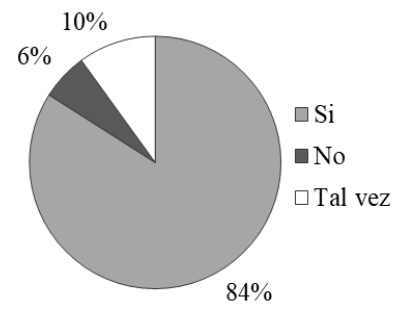

Fuente: Elaboración del autor (2020). 
Finalmente, la Figura 2 presenta el gráfico cajas y bigotes para la calificación del proceso editorial del JBC. El promedio fue de 7,48, el intervalo de calificación estuvo entre 6 y 9 con datos atípicos de 4, 5 y 10. Estos resultados indican un procedimiento editorial adecuado pero debe ser mejorado para obtener mejores indicadores.

Figura 2 - Gráfico de cajas y bigotes de la calificación al proceso editorial del JBC

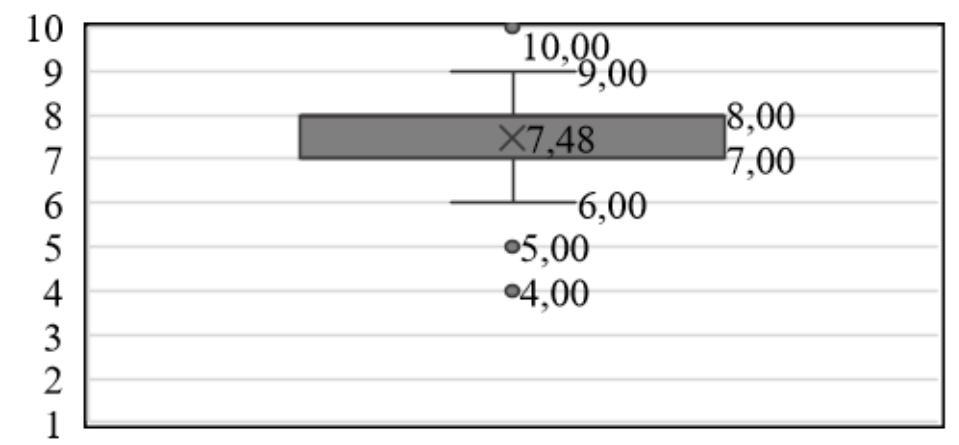

Fuente: Elaboración del autor (2020).

\section{Consideraciones finales}

El JBC debe mejorar en la visibilidad internacional, indexación y rapidez en el proceso de revisión por pares. Aspectos primordiales en la elección de una revista científica según los autores. Si bien el JBC se ha reestructurado en los últimos años, las características mencionadas deben ser esenciales en los lineamientos de la revista en los próximos años. A pesar que la encuesta se limitó a los autores de los últimos números publicados, la retroalimentación recibida permite identificar los puntos débiles de la revista y generar espacios para la mejora del proceso editorial.

\section{Referências}

VRANA, R. Editorial challenges in a small scientific community: Study of Croatian editors. Learned Publishing, 2018. DOI: https://doi.org/10.1002/leap.1188

HAUSMANN, L.; MURPHY, S. P. The challenges for scientific publishing, 60 years on. Journal of Neurochemistry, n.139, 280-287, 2016. DOI: https://doi.org/10.1111/jnc.13550

MARGALIDA, A; COLOMER, M. Improving the peer-review process and editorial quality: key errors escaping the review and editorial process in top scientific journals. PeerJ, v.4, n.1670, 2016. DOI: https://doi.org/10.7717/peerj.1670

XIE, Y.; WU, Q.; LI, X. Editorial team scholarly index (ETSI): an alternative indicator for evaluating academic journal reputation. Scientometrics, 2019. DOI: https://doi.org/10.1007/s11192$\underline{019-03177-\mathrm{x}}$ 
BESPALOV, A.; STECKLER, T.; SKOLNICK, P. Be positive about negatives-recommendations for the publication of negative (or null) results. European Neuropsychopharmacology, 2019. DOI: https://doi.org/10.1016/j.euroneuro.2019.10.007

${ }^{1}$ Joaquin Humberto Aquino Rocha (Tiquipaya, Cochabamba, Bolivia)
Graduado en Ingeniería Civil por la Universidad Mayor de San Simón - UMSS (Bolivia). Maestría en Ingeniería
Civil por la Universidad de Pernambuco - UPE (Brasil). Coordinador de investigación en Tecnología de la
Universidad Privada del Valle y editor de sección en Ingeniería Civil del Journal Boliviano de Ciencias.
Contribución de autoría: Conceptualización, Curación de datos, Análisis formal, Supervisión, Validación,
Visualización, Redacción (borrador original) y Redacción (revisión y edición).
Lattes: http://lattes.cnpq.br/1787659871780888
E-mail: joaquinaquinorocha@gmail.com

${ }^{2}$ Edson Gastón Montaño Bautista (Tiquipaya, Cochabamba, Bolivia)

Graduado en Ingeniería Mecánica y de Automatización Industrial por la Universidad Privada del Valle (Bolivia). Coordinador del Departamento Académico de Electromecánica de la Universidad Privada del Valle y editor de sección de Electromecánica del Journal Boliviano de Ciencias.

Contribución de autoría: Investigación, Administración del proyecto, Recursos, Visualización, Redacción (borrador original) y Redacción (revisión y edición).

E-mail: $\underline{\text { mbautistaedson@ gmail.com }}$

\section{${ }^{3}$ Daysi Lidia Iñiguez Calveti (Tiquipaya, Cochabamba, Bolivia)}

Graduada en Ingeniería Industrial por la Universidad Mayor de San Simón - UMSS (Bolivia). Maestría en Dirección de Proyectos de la Universidad Rey Juan Carlos (España). Coordinadora del Departamento de Industrias de la Universidad Privada del Valle y editora de sección en Industrias y Alimentos del Journal Boliviano de Ciencias.

Contribución de autoría: Investigación, Administración del proyecto, Recursos, Visualización, Redacción (borrador original) y Redacción (revisión y edición).

E-mail: diniguez@univalle.edu

Avaliador(a) responsável:

\section{Como citar este artigo:}

ROCHA, Joaquin Humberto Aquino, MONTAÑO-BAUTISTA, Edson Gastón, IÑIGUEZCALVETI, Daysi Lidia. Acciones de mejora en el proceso editorial científico del Journal Boliviano de Ciencias: estudio de caso. In: ABEC Meeting Live, 2020. Anais... São Paulo: Associação Brasileira de Editores Científicos, 2020. DOI: http://dx.doi.org/10.21452/abecmeeting2020.01 